\title{
Reflections on Using Chat-Based Platforms for Online Interviews with Screen-Reader Users
}

\author{
Rachel Menzies \\ r.menzies@dundee.ac.uk \\ University of Dundee
}

Dundee

\author{
Benjamin M. Gorman \\ bgorman@bournemouth.ac.uk \\ Bournemouth University \\ Bournemouth
}

\author{
Garreth W. Tigwell \\ garreth.w.tigwell@rit.edu \\ School of Information \\ Rochester Institute of Technology \\ Rochester, NY
}

\begin{abstract}
Within accessibility research, it is important for researchers to understand the lived experience of participants. Researchers often use in-person interviews to collect this data. However, in-person interviews can result in communication barriers and introduce logistical challenges surrounding scheduling and geographical location. For a recent study involving screen reader users, we used online chatbased platforms to conduct interviews. Unlike in-person interviews, there was little guidance within the field on conducting interviews using these platforms with screen reader users. To understand how effective these platforms were, we collected feedback from our participants on their experience after completing their interview. In this paper, we report on our experience of conducting online chatbased interviews with screen reader users. We present reflections from both the interviewer and participants on their experiences during the aforementioned study, and outline four lessons we learned during the process.
\end{abstract}

\section{CCS CONCEPTS}

\section{- Human-centered computing $\rightarrow$ Accessibility.}

\section{KEYWORDS}

Accessibility, Methodology

\section{ACM Reference Format:}

Rachel Menzies, Benjamin M. Gorman, and Garreth W. Tigwell. 2020. Reflections on Using Chat-Based Platforms for Online Interviews with ScreenReader Users. In The 22nd International ACM SIGACCESS Conference on Computers and Accessibility (ASSETS '20), October 26-28, 2020, Virtual Event, Greece. ACM, New York, NY, USA, 4 pages. https://doi.org/10.1145/3373625. 3418000

\section{INTRODUCTION \& BACKGROUND}

Interviews are a popular data collection approach within $\mathrm{HCI}$ research, and were the third highest reported method within accepted papers at CHI 2014 [2]. A primary advantage of interviews are the deep participant insights that can be gained where other methods fall short [7]. Including people with disabilities in research is important [10], and interviews allow researchers to understand the lived experience of disabled people.

Permission to make digital or hard copies of part or all of this work for personal or classroom use is granted without fee provided that copies are not made or distributed for profit or commercial advantage and that copies bear this notice and the full citation on the first page. Copyrights for third-party components of this work must be honored.

For all other uses, contact the owner/author(s).

ASSETS '20, October 26-28, 2020, Virtual Event, Greece

(C) 2020 Copyright held by the owner/author(s).

ACM ISBN 978-1-4503-7103-2/20/10

https://doi.org/10.1145/3373625.3418000
Although rewarding, in-person interviews are a time consuming data collection approach [7], and introduce other logistical challenges. Since they are a synchronous data collection method, they require thoughtful scheduling. Participants or researchers typically need to travel to a lab or other mutually convenient location, and as such interviews often take place in public spaces. Therefore, for in-person interviews participants are typically only able to be sourced within the same geographical location. Furthermore, global events such as COVID-19 can introduce additional constraints on conducting in-person research due to social distancing protocols.

Technology can minimize logistical and geographical challenges of in-person interviews [6] and online interviews have gained more attention [9]. Video calls can mimic in-person interviews [5], but are susceptible to poor internet connections. Email and instant messaging are also possible $[1,8,12,16]$, and these chat-based interviews provide more privacy, are asynchronous, and self-transcribed saving researchers time [6]. It is important to note that instant messaging can still result in rich data, even if fewer words are shared than telephone or in-person interviews $[3,11]$.

Recruiting participants for accessibility research is challenging [10] and internet-based qualitative approaches can support research with hard-to-reach populations [15]. Although prior work discusses the advantages and disadvantages of such different research approaches, there is no guidance on using chat-based platforms with screen-reader users.

\subsection{Study Context \& Contributions}

In our recent $\mathrm{CHI}$ Paper [13], we investigated how accessible emoji are for visually impaired people. We used online chat-based platforms, e.g. Facebook Messenger, to interview screen reader users. Online chat interviews were more convenient (e.g., reducing the need to find somewhere private) and emoji could be shared easily. Participants chose the platform to ensure accessibility. In this paper, we report on our experience of conducting online chat-based interviews with screen reader users. This paper makes two contributions: 1) We reflect on participant and interviewer experience of synchronous online interview using chat based platforms with screen-reader users. 2) We provide lessons learned through conducting the interviews, which may help future researchers when using this approach.

\section{PARTICIPANT REFLECTIONS}

After our online interviews, we invited participants to complete an online questionnaire addressing the research question: What was the experience of screen-reader users using chat-based platforms for online interviews? 
Nine participants completed the questionnaire $($ Male $=6$; Female $=2$; Agender=1), aged between $18-37$ years old $(M=27.63, S D=6.72)$. Participants rated their visual acuity using descriptions proposed by the World Health Organisation [4]: "Severe (worse than 20/200 and equal to or better than 20/400" (1 participant), and "Blind (worse than 20/400)", (7 participants). One participant responded: "Full blindness with zero light perception but physical eyes still remain".

\subsection{Method}

Our online questionnaire had 18 questions (12 closed-ended and six open-ended). We used the first five questions to understand what prior experience participants had with research studies (Q1), using online chat (Q2) and the purpose (Q3), whether the interview length was sufficient (Q4) and to comment on the reason for their response (Q5). Q6-14 included nine statements shown in Table 1 and we presented the statements with a corresponding five point Likert scale. We concluded the interview with broader questions to find out the advantages $(\mathrm{Q} 15)$ and disadvantages (Q16) the participants identified, any advice participants had for researchers conducting chat-based with screen-reader users (Q17), and final comments on the platform each participant chose (Q18). Ethical approval was obtained from our IRB. The participants were reimbursed for their time with an Amazon voucher equivalent to $£ 15$.

\subsection{Quantitative Findings}

Prior to this study, participants had varied previous experience of using online chat platforms. Three participants had used online chat to speak to a business at least once per day, three did this less than once per month and 3 had never done this. Participants provided examples of engaging in online chat and these were varied: home broadband, a phone company, troubleshooting with Google, flight carriers, energy supplier. Of the participants who reported that they never use online chat, one provided a valid example (suggesting that this response was in error, one noted that they have engaged in online chat for personal use, and one noted that they "avoid these services because they tend to have accessibility problems". Participants were asked a number of statements on a 5-point Likert scale (Strongly Disagree - Disagree - Neutral - Agree - Strongly Agree). These results are shown in Table 1. Participants were also asked whether the length of the interview was sufficient to cover the questions posed. All participants responded Yes.

\subsection{Qualitative Findings}

An open-coding approach was used by the primary author to analyse the qualitative responses [14]. Initial axial codes were generated using a data-driven approach and then collated. We identified three thematic sections, outlined below.

1) The Study Environment: In terms of the physical location, participants commented that they "don't have to worry about noisy locations" (P4) or "disrupting others" (P2), suggesting chat-based platforms added flexibility. P1 reported they would be "able to do a text chat interview almost anywhere at almost any time". Concerning the digital environment, $\mathrm{P} 4$ noted that text "consumes smaller bandwidth than a voice chat". There were reports of potential technical limitations, e.g., "a disturbingly large portion of blind people still use Internet Explorer, and compatibility with many web clients could be patchy or even non existent" (P1). However, all participants had a choice of chat platform.

2) Conversational Interaction: The speed of conversation was reduced, with participants noting "it could take screen readers longer to read and respond to the chat" (P10). P1 reported challenges when receiving a message: "this is even worse for a screen reader user because we can't just glance at it and skim read, we have to exit the text box, arrow back up, then reenter the text box and find where we left off". However, the slower pace eased communication, allowing participants to "have the time to think (P10) as well as "check older messages, which is less embarrassing than admitting you lost track and therefore much more likely to actually be effective" (P1).

Regarding the inter-personal connection with the interviewer, P1 noted that the text-based chat was "less personable". Preferences on the most natural interaction form varied. P8 found typing "more natural" whereas P11 noted "sometimes saying what you want to say is easier". Some limitations of text were identified. Although it was possible for participants to send a variety of media content via the chat, most participants sent only text. It was noted that some people "cannot write their thoughts into words and express themselves" (P4) and that "getting all your thoughts in one or two messages without creating a mind numbing wall of text is hard for some" (P1).

3) Convenience: The chat functionality complemented data gathering, resulting in an "easily-archived record is also available" (P8) after the interview. There was also a convenience in participant preparation of their answers. Participants can "take some time to think about the questions without pressure" (P3) and this means that "information may end up being more coherent and better able to be parsed into usable data" (P1).

\section{INTERVIEWER REFLECTIONS}

The primary author conducted interviews across different platforms: Facebook Messenger, Twitter DM and Google Hangout. The platform for each interview was chosen by the participant to ensure that they were comfortable with the system and any additional Assistive Technology required. Facebook was by far the most requested system, most likely due to participants using this system regularly. It was important that privacy was considered to ensure that the two parties did not need to become 'friends'. Therefore, accounts were set up on these platforms to be used for this study.

In a face-to-face interview, active listening plays a large role (e.g., nodding). During the chat-based interview, this was provided using verbal acknowledgment responses (e.g. 'yes, I understand'). These responses were typically announced by screen-readers and so were disruptive for participants. Conversation did not flow as readily as a voice conversation.

There was no icon to indicate when participants were typing. This shows on most messaging platforms and reassures the conversation partner that a response is imminent. However, because we used a Facebook Page to maintain privacy, the 'typing' icon was not present. Some participants answered questions by typing one long response. For the interviewer, this meant that responses took a long time to arrive and it was not clear that the participant was still engaged. Second, some participants typed an answer in one 
Table 1: Summary of participant quantitative responses to questions 6-14 of our questionnaire. No responses were given for Strongly Disagree or Disagree, so these have been omitted from the table.

\begin{tabular}{lccc}
\hline Statement & Neutral & Agree & Strongly Agree \\
\hline Q6. I was able to share the information I wanted to during the interview & - & 3 & 6 \\
Q7. The interview moved at a suitable pace & 1 & 3 & 5 \\
Q8. The interviewer was engaged & - & 3 & 6 \\
Q9. I was engaged with the interview & - & 2 & 7 \\
Q10. I was able to expand on points that I made during the interview & - & 2 & 7 \\
Q11. I felt comfortable during the interview & 1 & 3 & 5 \\
Q12. I enjoyed taking part in the online chat interview & - & 4 & 5 \\
Q13. As a screen-reader user, online chat interviews were a good way to & 1 & 6 & 2 \\
share my opinions and ideas & & & \\
Q14. I would recommend that others take part in online chat interviews & 1 & 4 & 4 \\
\hline
\end{tabular}

message with follow-up responses in subsequent messages. Without the icon being present, it was not clear when the response was complete. This sometimes led to the interviewer asking a question before the participant had completed their response, which needed additional effort to repair the conversation.

Within Facebook, a 'page' is linked to a personal account with individuals being administrators of the page. This means that in order to access the page, the interviewer was logged into their personal account. Notifications for personal accounts were a distraction at times, particularly when waiting for participants to construct lengthy replies.

\section{LESSONS LEARNED}

We have identified four lessons we learned that can inform future use of online chat-based interviews:

1) Consider if conversation acknowledgments are required (i.e., messages with similar intentions to showing active listening). Ensure that these do not interrupt screen-reader users when they are dictating or typing responses.

2) Screen reader users may have additional steps in replying within the conversation, such as dictating their response and reading it back for clarity, which reduced the interaction speed. Using a platform with an icon for 'typing' ensures that the interviewer can see that the participant is responding and avoid typing questions that may interrupt this flow.

3) We set up a Facebook page to avoid participants becoming 'friends'. However, the page must be linked to the interviewer's personal account, which introduced notification challenges. Using a page should have reduced distraction but personal notifications on Facebook could not be muted without also muting those from the page.

4) Scheduling time between participants is common in face-toface interviews and should also be used for chat-based interviews. This allows extra time in cases where the responses from screenreader users are slower than expected. In some cases, participants would message ahead of time to indicate that they were available and this would overlap with an interview in progress, adding to the notification distractions for the interviewer.

These lessons are based on the experience of both the interviewer and the research participants, who are screen reader users.
While we feel they will be useful when considering this method in general, they will be especially beneficial when interviewing participants who use screen-readers. We found great benefit in using this method, but there is more to learn and we would be interested to see how how generalizable our experiences are is to other participant groups.

\section{REFERENCES}

[1] Roberta Bampton and Christopher J Cowton. 2002. The e-interview. In Forum Qualitative Sozialforschung/Forum: Qualitative Social Research, Vol. 3. https: //doi.org/10.17169/fqs-3.2.848

[2] Kelly Caine. 2016. Local Standards for Sample Size at CHI. In Proceedings of the 2016 CHI Conference on Human Factors in Computing Systems (San Jose, California, USA) (CHI '16). ACM, New York, NY, USA, 981-992. https://doi.org/10.1145/ 2858036.2858498

[3] Jill P. Dimond, Casey Fiesler, Betsy DiSalvo, Jon Pelc, and Amy S. Bruckman. 2012. Qualitative Data Collection Technologies: A Comparison of Instant Messaging, Email, and Phone. In Proceedings of the 17th ACM International Conference on Supporting Group Work (Sanibel Island, Florida, USA) (GROUP '12). ACM, New York, NY, USA, 277-280. https://doi.org/10.1145/2389176.2389218

[4] World Health Organisation (WHO) Programme for the Prevention of Blindness and Deafness. 2003. Consultation on development of standards for characterization of vision loss and visual functioning. https://apps.who.int/iris/handle/10665/68601. Accessed: 2019-09-03.

[5] Roksana Janghorban, Robab Latifnejad Roudsari, and Ali Taghipour. 2014. Skype interviewing: The new generation of online synchronous interview in qualitative research. International fournal of Qualitative Studies on Health and Well-being 9, 1 (2014), 24152. https://doi.org/10.3402/qhw.v9.24152

[6] Michelle M. Kazmer and Bo Xie. 2008. QUALITATIVE INTERVIEWING IN INTERNET STUDIES: Playing with the media, playing with the method. Information, Communication \& Society 11, 2 (2008), 257-278. https://doi.org/10.1080/ 13691180801946333

[7] Jonathan Lazar, Jinjuan Heidi Feng, and Harry Hochheiser. 2017. Research methods in human-computer interaction. Morgan Kaufmann.

[8] Judith L. M. McCoyd and Toba Schwaber Kerson. 2006. Conducting Intensive Interviews Using Email: A Serendipitous Comparative Opportunity. Qualitative Social Work 5, 3 (2006), 389-406. https://doi.org/10.1177/1473325006067367

[9] Gina Novick. 2008. Is there a bias against telephone interviews in qualitative research? Research in Nursing \& Health 31, 4 (2008), 391-398. https://doi.org/10. 1002/nur.20259 arXiv:https://onlinelibrary.wiley.com/doi/pdf/10.1002/nur.20259

[10] Andrew Sears and Vicki L. Hanson. 2012. Representing Users in Accessibility Research. ACM Trans. Access. Comput. 4, 2, Article 7 (March 2012), 6 pages. https://doi.org/10.1145/2141943.2141945

[11] Jennifer D. Shapka, Jose F. Domene, Shereen Khan, and Leigh Mijin Yang. 2016. Online versus in-person interviews with adolescents: An exploration of data equivalence. Computers in Human Behavior 58 (2016), 361 - 367. https://doi.org/ 10.1016/j.chb.2016.01.016

[12] Stefan Stieger and Anja S. Göritz. 2006. Using Instant Messaging for InternetBased Interviews. CyberPsychology \& Behavior 9, 5 (2006), 552-559. https: //doi.org/10.1089/cpb.2006.9.552 
[13] Garreth W. Tigwell, Benjamin M. Gorman, and Rachel Menzies. 2020. Emoji Accessibility for Visually Impaired People. In Proceedings of the 2020 CHI Conference on Human Factors in Computing Systems (Honolulu, HI, USA) (CHI '20). Association for Computing Machinery, New York, NY, USA, 1-14. https: //doi.org/10.1145/3313831.3376267

[14] Sarah J. Tracey. 2013. Qualitative Research Methods: Collecting Evidence, Crafting Analysis. Wiley-Blackwell.
[15] J. Michael Wilkerson, Alex Iantaffi, Jeremy A. Grey, Walter O. Bockting, and B. R. Simon Rosser. 2014. Recommendations for Internet-Based Qualitative Health Research With Hard-to-Reach Populations. Qualitative Health Research 24, 4 (2014), 561-574. https://doi.org/10.1177/1049732314524635

[16] Jason Zalinger, Nathan Freier, and Eric Dutko. 2009. Ethnochat: An Instant Messenger Program for Ethnography. In CHI '09 Extended Abstracts on Human Factors in Computing Systems (Boston, MA, USA) (CHI EA '09). ACM, New York, NY, USA, 3703-3708. https://doi.org/10.1145/1520340.1520558 